\title{
Reflections on the US FDA's Warning on Direct-to-Consumer Genetic Testing
}

\author{
Seon-Hee Yim $^{1 *}$, Yeun-Jun Chung ${ }^{2 * *}$ \\ ${ }^{1}$ Department of Medical Education, Integrated Research Center for Genome Polymorphism, \\ MRC, College of Medicine, The Catholic University of Korea, Seoul 137-701, Korea, \\ ${ }^{2}$ Department of Microbiology, Integrated Research Center for Genome Polymorphism, \\ MRC, College of Medicine, The Catholic University of Korea, Seoul 137-701, Korea
}

In November 2013, the US Food and Drug Administration (FDA) sent a warning letter to 23andMe, Inc. and ordered the company to discontinue marketing of the 23andMe Personal Genome Service (PGS) until it receives FDA marketing authorization for the device. The FDA considers the PGS as an unclassified medical device, which requires premarket approval or de novo classification. Opponents of the FDA's action expressed their concerns, saying that the FDA is overcautious and paternalistic, which violates consumers' rights and might stifle the consumer genomics field itself, and insisted that the agency should not restrict direct-to-consumer (DTC) genomic testing without empirical evidence of harm. Proponents support the agency's action as protection of consumers from potentially invalid and almost useless information. This action was also significant, since it reflected the FDA's attitude towards medical application of next-generation sequencing techniques. In this review, we followed up on the FDA-23andMe incident and evaluated the problems and prospects for DTC genetic testing.

Keywords: direct-to-consumer genetic testing, medical device, regulation

\section{Introduction}

On November 22, 2013, the US Food and Drug Administration (FDA) sent a warning letter to Ann Wojcicki, co-founder and CEO of 23andMe, Inc. and ordered the company to "immediately discontinue marketing of the 23andMe Saliva Collection Kit and Personal Genome Service (PGS) until such time as it receives FDA marketing authorization for the device" [1]. Since 23andMe is one of the largest direct-to-consumer (DTC) genetic testing companies leading the field, the FDA's action could have big implications for the present and future. This FDA letter was even called a "bombshell" in one Nature Editorial [2]. Various responses and speculation have followed. Some people support the FDA's action as protection of consumers from potentially invalid information, while others insist that the FDA's action violates the rights of individuals to receive information and of 'commercial speech' and undermines democratization of health care and patient empowerment [3]. One of those responses is noteworthy: 7 months after the FDA's action, in June 2014, Lee and Lee [4] of the Korea Health Industry Development Institute (KHIDI) submitted a report to the Public-Private Joint Regulatory Advancement Initiative (PPJRAI), titled "Market Analysis and Strategies for Regulatory Reform in Medical Industry," which expressed a stance somehow different from that of the US FDA. To facilitate the discussion in our scientific community, we examined the development of the 23andMe event and reflected on the future of genetic-genome testing, including whole-genome sequencing as a medical device.

\section{A Quick Glance at DTC Genetic Testing and 23andMe}

DTC genetic testing, also known as at-home genetic 
testing, refers to genetic tests that are sold directly to consumers via the internet, television, or print advertisement without necessarily involving health care providers. Su [5] grouped the reasons for using DTC genetic tests into three categories; identity (such as ancestry, paternity, or ethnicity)-seeking; disease risk-testing to complement health care; and curiosity-driven testing. DTC genetic testing has its pros and cons. It is more accessible and affordable than traditional genetic tests, which usually target one specific gene at a time. DTC testing companies and many personalized medicine advocates argue that knowing the genetic risks for potential diseases will help people to improve their health and wellness by changing what they can and managing what they can not, as claimed in 23andMe's TV commercial [6]. 23andMe, one of the largest DTC genetic testing companies, also made the same claims and has offered the PGS to $>475,000$ customers since 2007 [7]. 23andMe's PGS genotypes the DNA sample on their custom single-nucleotide polymorphism (SNP) chip and provides health reports on 254 diseases and traits, including carrier status and drug response, which, the company claims, enables customers to personalize their health care. Additionally, some proponents suggest, as another argument, that DTC genetic testing can contribute to the empowerment of consumers by increasing knowledge and to subsequent democratization of health care [5].

However, many geneticists and physicians have rejected the claims. They believe that since DTC genetic testing is not reliable enough to be used clinically, it will just end up increasing health care costs unnecessarily [8]. Apart from its scientific weaknesses and insufficient clinical utility, there are other concerns raised by critics, including psychological and financial damage to consumers, especially by unreliable test results, health care inequity, and genetic privacy.

Since we believe that scientific validity is the foremost foundation of DTC genetic testing and should presuppose its clinical usage, we will focus on it in this review. There are quite a few scientific limitations to genome-based health risk estimation services in general, not just 23andMe's PGS, but they may not be apparent, even to medical professionals, who do not have enough knowledge in genetics and genomics, as well as lay customers. First of all, for monogenic disorders, DTC genetic testing panels include a small fraction of known disease-causing mutations or sometimes just include mutations present only in specific populations. This incompleteness of testing results in misinterpretations, which makes its clinical value doubtful. Also, for polygenicmultifactorial disorders and conditions, DTC testing panels include only a small percentage of the markers among many known associated markers, not to mention unknown ones, which also makes the risk prediction incomplete and, as a result, inaccurate. We also should not forget that gene-gene and gene-environment interactions are not taken into consideration for risk estimation at all. In addition, it is hard to say that their algorithms for estimating total combined risks are very well validated. In summary, genome-based DTC tests are incomplete, and the interpretation of their results is hardly valid. The biology is much more complicated than some health care entrepreneurs believe, and genotypes and sequences are not information but are just basic tools to use for understanding biology. Even in the case of valid associations that have been replicated by many studies, the effect sizes of most SNPs on specific diseases seem to be small to modest [9]. Secondly, even though their customers are provided detailed background information on the method used for genotyping and the statistical interpretation, which supposedly helps their understanding, there is still ample possibility for misconceptions.

Some professional societies have issued statements on DTC genetic testing. The American College of Medicine Genetics said, in their 2003 statement, that due to the complexities of testing and counseling, patients' use of DTC genetic testing kits may pose harms, which include the following: test utilization for inappropriate purposes, misinterpretation of test results, and lack of necessary follow-up [10]. In 2007, the American Society of Human Genetics also expressed their concerns in a statement on DTC genetic testing, saying that consumers are at risk of harm if the quality of tests is not high enough-i.e., the analytical or clinical validity and clinical utility of tests are not appropriate [11]. Analytical validity shows how accurately and reliably the test detects a specific genetic variant. Clinical validity shows how well the genetic variant is related to the existence or risk of a specific disease or condition. Clinical utility shows how likely the test can improve patient outcomes [12]. Consumers are also at risk of harm if claims made about tests are misleading or simply false and if information and counseling are not properly provided to enable the consumer to make an informed decision about whether to take the test or not and about what actions to take after the genetic test [11]. Due to these criticisms and repeated interventions from regulators, including the US FDA, especially in the United States, the DTC market does not look very promising at this moment. Even one Nature Editorial said that DTC genetic testing is not yet a viable business model, and most firms in the market have stopped providing services involved with personal disease risk estimation or have even totally shut down [2].

\section{FDA's Action}

On November 22, 2013, the US FDA sent a warning letter 
to 23 andMe, Inc. and ordered the company to immediately cease marketing of their Saliva Collection Kit and PGS until it received proper marketing authorization for the device [1]. This letter was sent after the firm failed to provide additional information on the analytical and clinical validity of the PGS the FDA requested. It said that although the FDA has been diligently working to help the company comply with regulatory requirements since July 2009, it stopped communicating with the FDA since May. Apparently making matters worse, the company initiated new marketing campaigns, including TV commercials. This made the FDA conclude that the company intends to expand the PGS's uses and consumer base without obtaining marketing authorization from the agency [2]. The FDA clearly stated that their PGS requires marketing approval, because it is intended for use "in the diagnosis of disease or other conditions or in the cure, mitigation, treatment, or prevention of disease, or is intended to affect the structure or function of the body." In other words, the FDA considers the PGS an unclassified medical device, which requires premarket approval or de novo classification [1].

After receiving the letter, on November 26, 2014, Wojcicki acknowledged in a blog post that they were behind schedule with their responses to the FDA and that the agency needs to be convinced of the quality of their PGS data. On December 5,2013 , the company announced that it would suspend its health-related genetic tests to comply with the US FDA's directive during the regulatory review process [13]. Based on the letter, the FDA does not seem to think that the analytical validity of 23andMe's SNP genotyping is a problem per se as far as its results are correct but feels that the clinical validity of risk estimation has yet to be validated, which may be practically impossible. After that, 23andMe announced that it has been working with the FDA, and the agency seemed to consider the firm's response to be adequate [14]. However, the firm is not able to provide any timeline for resuming their PGS.

\section{Various Reactions}

The US FDA's action evoked mixed reactions. Some expressed their concerns, saying that the FDA is overcautious and paternalistic and hinders medical advances. According to Green and Farahany [3], the FDA's action violates the rights of individuals to receive information and of 'commercial speech' and undermines the democratization of health care and patient empowerment. Also, they were concerned that this kind of overcautious regulation might stifle the consumer genomics field itself and insisted that the agency should not restrict DTC genomic testing without empirical evidence of harm. However, rather ironically, they ended up emphasizing the PGS's poor clinical utility by saying that health-related interpretations of the PGS, particularly about common genetic variants, relate only indirectly to preventing or diagnosing disease [4]. If their comment is true, what is the point of taking the test? It is simply not ethical to entice people to pay for useless products. One non-profit technology policy think tank, called TechFreedom, launched an online petition asking the FDA to reverse the ban and collected a total of 10,073 signatures on January 18, 2014 [15]. Many petitioners said that their genetic codes belong to them, so they have the "right to know" what is written there, and that the assumption that they should be "protected from knowing" because they are not smart enough to understand is simply insulting. All of these concerns are understandable, but we insist that genetic testing with scientific flaws itself is a great harm to consumers and that those flaws need to be addressed before discussing the protection of anyone's right to know or not to know.

Of course, there are many people who support the FDA's action as protection of consumers from potentially invalid and almost useless information. Among the professional proponents, Baudhuin emphatically wrote in her opinion paper that if it is too burdensome to prove the validity of the information that the DTC testing companies offer, they should not sell the information in the first place [2]. Some consumers are already initiating procedures for class-action lawsuits against 23andMe, claiming that it is selling misleading information [2]. On November 27, 2013, just 5 days after the FDA's warning letter was sent to 23andMe, the first class-action lawsuit was already filed in the Southern District Court of California [16]. However, even among the supporters, some people saw the FDA's action as rather unusual, because the decision was largely based on its theoretical harm rather than actual harm, which the FDA has usually been waiting for before acting.

In Korea, Lee and Lee [4] of the KHIDI submitted a report in June 2014 to the PPJRAI, titled "Market Analysis and Strategies for Regulatory Reform in Medical Industry," which expressed a stance somehow different from that of the US FDA [4]. In this report, they suggested that it will help to expand the PGS market if genetic testing agencies are exempted from providing at least two scientific reports to support or prove the clinical validity of their tests when they make a notification of their testing items for disease prevention to the Korea Center for Disease Control and Prevention [4]. This recommendation seems to be in opposition to the US FDA's. Also, they recommended an amendment to the Bioethics and Safety Act, which will allow non-medical institutions, such as biotechnology companies, to perform health-related PGSs, at least for disease predic- 
tion and prevention (not diagnosis) [4]. However, they added that this predictive, preventive genetic testing should be performed under the supervision of physicians, like the US FDA insists. Since these recommendations are supposed be delivered to the ministries involved in regulating genetic testing, we may guess which direction our governmental regulation system will take in the near future.

23andMe is now working to get FDA approval for its health tests; the company submitted seven health-related tests to the FDA for review in July 2014; it plans to submit 100 additional tests before the end of the year [17]. However, it is very likely that this process will take years. However, they have been trying different strategies, including finding new markets. From October 2014, 23andMe has begun selling its health-related genetic tests in Canada, despite still being blocked by the US FDA from offering its health risk-related service [18].

\section{Future Implications of the US FDA's Action}

Downing and Ross wrote that sales of DTC genetic tests, including 23andMe's PGS, could be resumed only if reliable data have been accumulated that can demonstrate that the tests have positive clinical validity and can be used safely without professional supervision [19]. As we mentioned above, this task is easier said than done - it can be practically impossible. Even the same authors actually emphasized the importance of the physician's role in the same article, which can "contextualize" test results for maximizing clinical benefit, preventing adverse effects, and genuinely empowering patients [19]. Zettler et al. [20] wrote that the FDA's warning to 23andMe may effectively mark the end of the DTC genetic testing field, at least in the United States, if 23andMe's PGS cannot meet the FDA's standards for clinical validity. DTC genetic testing companies, such as Navigenics and deCODEme, were already sold to other firms and stopped their health-related services in 2012 [20]. The problem is that no one knows, at the moment, how strict the FDA's standards for clinical validity will be.

Another question will definitely be this: what will happen to whole-genome and exome sequencing, which is already replacing single-gene tests or SNP chips, in terms of clinical application? Interestingly, just 3 days before warning 23andMe, the FDA authorized Illumina to market four "next-generation" gene sequencing devices: a sequencing platform, two assays - the MiSeqDx Cystic Fibrosis 139Variant Assay and MiSeqDx Cystic Fibrosis Clinical Sequencing Assay - and a "universal kit" that allows laboratories that purchase the platform to develop and validate sequencing of any part of a patient's genome and to find any differences between the patient and the reference [21]. What made the difference in the fate of these two companies? We have not made an inquiry about this directly to the US FDA but can get some clues from the FDA's press release about this authorization. First of all, it said that these assays will check "specific points in the patient's CFTR gene sequence" to detect known and unknown variants. Their reports will include the existence of genetic variants in the gene sequence that are known to be associated with one specific disease but will not provide medical interpretations of the findings. Second, it did not mention non-patient customers at all. Finally, the press release also emphasized the clinician's involvement. It said that "this information-rich technology is becoming more accessible for use by physicians in the care of their patients" and that "the new technology also gives physicians the ability to take a broader look at their patients' genetic makeup and can help in diagnosing disease or identifying the cause of symptoms" [21]. We believe that the US FDA's authorization of the MiSeqDX for cystic fibrosis reflects the agency's attitude towards any tests involving the human genome. Therefore, from this, we can guess the answer to the question we posed above: any genetic/genome/exome tests will be required to prove their analytical and clinical validity and clinical utility for specific diseases and conditions if they are intended for medical applications. Until then, we suggest that their application should be restricted to research applications and family studies for diagnosing genetic disorders of unknown etiology. When a human being is involved, we believe that the most important ethics principle is "Do no harm."

\section{References}

1. US Food and Drug Administration. Warning letter, Nov 22, 2013. Silver Spring: US Food and Drug Administration, 2013. Accessed 2014 Oct 31. Available from: http://www.fda.gov/ iceci/enforcementactions/warningletters/2013/ucm376296. htm.

2. The FDA and me. Nature 2013;504:7-8.

3. Green RC, Farahany NA. Regulation: the FDA is overcautious on consumer genomics. Nature 2014;505:286-287.

4. Lee KL, Lee SM. Market Analysis and Strategies for Regulatory Reform in Medical Industry. Public-Private Joint Regulation Advancement Initiative, 2014.

5. Su P. Direct-to-consumer genetic testing: a comprehensive view. Yale J Biol Med 2013;86:359-365.

6. iSpot.tv. 23 and Me (TV commercial). Bellevue: iSpot.tv, 2013. Accessed 2014 Nov 1. Available from: http://www.ispot.tv/ad/7qoF/23-and-me.

7. Wojcicki A. Just the facts, please. Nat Biotechnol 2013;31: 1075-1076.

8. Hogarth S, Javitt G, Melzer D. The current landscape for direct-to-consumer genetic testing: legal, ethical, and policy issues. Annu Rev Genomics Hum Genet 2008;9:161-182. 
9. Hindorff LA, Sethupathy P, Junkins HA, Ramos EM, Mehta JP, Collins FS, et al. Potential etiologic and functional implications of genome-wide association loci for human diseases and traits. Proc Natl Acad Sci U S A 2009;106:9362-9367.

10. American College of Medicine Genetics Board of Directors. ACMG statement on direct-to-consumer genetic testing. Genet Med 2004;6:60.

11. Hudson K, Javitt G, Burke W, Byers P; American Society of Human Genetics Social Issues Committee. ASHG Statement* on direct-to-consumer genetic testing in the United States. Obstet Gynecol 2007;110:1392-1395.

12. US Centers for Disease Control and Prevention. Genomic testing: ACCE model process for evaluating genetic tests. Atlanta: US Centers for Disease Control and Prevention, 2010. Accessed 2014 Nov 1. Available from: http://www. cdc.gov/genomics/gtesting/ACCE/.

13. 23andMe. FDA update: December 5th. Palo Alto: 23andMe, 2013. Accessed 2014 Nov 1. Available from: https:// customercare.23andme.com/entries/23273619-FDAUpdate-December-23273615th.

14. US Food and Drug Administration. Close out letter, March 25, 2014. Silver Spring: US Food and Drug Administration, 2014. Accessed 2014 Nov 1. Available from: http://www.fda.gov/ ICECI/EnforcementActions/WarningLetters/ucm391016.htm.

15. TechFreedom. FDA: Don't ban marketing of home genomics kits like 23andMe. Change.org, 2014. Accessed 2014 Nov 1.
Available from: https://www.change.org/petitions/fda-dont-ban-marketing-of-home-genomics-kits-like-23andme.

16. O'Connor L. 23andMe faces $\$ 5$ million lawsuit days after being banned by FDA. The Huffington Post, 2013. Accessed 2014 Nov 1. Available from: http://www.huffingtonpost. com/2013/2012/2004/2023andme-lawsuit_n_4387699.html.

17. CBS News. 23andMe personalized DNA test seeks FDA approval. New York: CBS Interactive Inc., 2012. Accessed 2014 Nov 1. Available from: http://www.cbsnews.com/ news/23andme-personalized-dna-test-seeks-fda-approval/.

18. CBC News. 23andMe genetic testing service raises ethical questions. New York: CBS Interactive Inc., 2014. Accessed 2014 Nov 1. Available from: http://www.cbc.ca/news/business/23andme-genetic-testing-service-raises-ethical-questions1.2785522 .

19. Downing NS, Ross JS. Innovation, risk, and patient empowerment: the FDA-mandated withdrawal of 23andMe's Personal Genome Service. JAMA 2014;311:793-794.

20. Zettler PJ, Sherkow JS, Greely HT. 23andMe, the Food and Drug Administration, and the future of genetic testing. JAMA Intern Med 2014;174:493-494.

21. US Food and Drug Administration. FDA allows marketing of four "next generation" gene sequencing devices. Silver Spring: US Food and Drug Administration, 2013. Accessed 2014 Nov 1. Available from: http://www.fda.gov/newsevents/newsroom/pressannouncements/ucm375742.htm. 